\title{
Rotating Charge Coupled to the Maxwell Field: Scattering Theory and Adiabatic Limit
}

\author{
By \\ Valery Imaikin $^{1, \dagger}$, Alexander Komech ${ }^{1, \ddagger}$, and Herbert Spohn ${ }^{2}$ \\ ${ }^{1}$ University of Vienna, Austria \\ ${ }^{2}$ TU München, Germany \\ Communicated by P. Markowich
}

Received July 24, 2002; in revised form October 8, 2003

Published online March 29, 2004 (C) Springer-Verlag 2004

\begin{abstract}
We consider a spinning charge coupled to the Maxwell field. Through the appropriate symmetry in the initial conditions the charge remains at rest. We establish that any time-dependent finite energy solution converges to a sum of a soliton wave and an outgoing free wave. The convergence holds in global energy norm. Under a small constant external magnetic field the soliton manifold is stable in local energy seminorms and the evolution of the angular velocity is guided by an effective finitedimensional dynamics. The proof uses a non-autonomous integral inequality method.
\end{abstract}

2000 Mathematics Subject Classification: 37K40

Key words: Spinning charge coupled to Maxwell field, soliton-type asymptotics, scattering, adiabatic limit

\section{Introduction}

Over the past years we have studied the dynamical properties of the Abraham model for a classical charge coupled to the Maxwell field, $[6,8,11,9]$. The Abraham model is semirelativistic, since the charge distribution is rigid and independent of the velocity. Although widely used, physically this model is not quite consistent: a rigid charged body which has zero internal angular momentum initially, will start to rotate, in general, through the self-generated Maxwell field. In this note we will remedy such a short-coming and study a few dynamical aspects of the Abraham model with spin.

The equations of motion are easily written down. The Maxwell field consists of the electric field $E(x, t)$ and the magnetic field $B(x, t)$. The charge has the center of mass $q$ with the velocity $\dot{q}$. For notational simplicity we assume that the mass distribution, $m \rho(x)$, and the charge distribution, $e \rho(x)$, are proportional to each

\footnotetext{
${ }^{\dagger}$ Supported partly by the Wittgenstein 2000 Award of Peter Markowich, funded by the Austrian Science Foundation (FWF), research grants of DFG (436 RUS 113/615/0-1(R)) and RFBR (01-0104002).

$\ddagger$ On leave Department of Mechanics and Mathematics, Moscow State University, Moscow 119899, Russia. Supported partly by Max Planck Institute for the Mathematics in Sciences (Leipzig) and the Austrian Science Foundation (FWF) START Project (Y-137-TEC) of Norbert Mauser.
} 
other. Here $m$ is the total mass, $e$ is the total charge, and $\rho(x)$ is a "smooth" radially symmetric smearing function of compact support, explicitly,

$$
\nabla \rho, \rho \in L^{2}\left(\mathbb{R}^{3}\right), \quad \rho(x)=\rho_{r}(|x|), \quad \rho(x)=0 \quad \text { for }|x| \geqslant R_{\rho}, \quad \int d^{3} x \rho(x)=1 .
$$

The angular velocity of the charge is denoted by $\omega(t) \in \mathbb{R}^{3}$. Then the Maxwell equations read (cf. $[4,11]$ and compare with $[1,2])$

$$
\dot{E}=\operatorname{rot} B-e(\dot{q}+\omega \wedge(x-q)) \rho(x-q), \quad \dot{B}=-\operatorname{rot} E,
$$

where the current has now a contribution also from the internal rotation, together with the constraints,

$$
\operatorname{div} E(x, t)=e \rho(x-q(t)), \quad \operatorname{div} B(x, t)=0 .
$$

The back reaction of the field onto the charge is given through the Lorentz force equation

$$
m \ddot{q}=e \int[E+(\dot{q}+\omega \wedge(x-q)) \wedge B] \rho(x-q) d^{3} x
$$

and the Lorentz torque equation

$$
I_{\mathrm{b}} \dot{\omega}=e \int(x-q) \wedge[E+(\dot{q}+\omega \wedge(x-q)) \wedge B] \rho(x-q) d^{3} x,
$$

with the moment of inertia

$$
I_{\mathrm{b}}=\frac{2}{3} m \int x^{2} \rho(x) d^{3} x
$$

If necessary, one could add external fields $E_{\mathrm{ex}}, B_{\mathrm{ex}}$ according to the standard rules.

The perhaps most basic question is to obtain solutions having constant velocity and being of the form

$$
q(t)=q+v t, \quad \omega(t) \equiv \omega, \quad E(x, t)=E_{v, \omega}(x-v t), \quad B(x, t)=B_{v, \omega}(x-v t) .
$$

If in (1.1), (1.3), (1.4) we set $\omega=0$, by hand, then for every $v \in \mathbb{R}^{3}$ there is a unique solution of the form (1.6). However, for the Abraham model including spin, the Eq. (1.4) can be satisfied only if either $\omega \| v$ or $\omega \perp v$ [10]. This result is surprising at first sight, but reflects the semirelativistic nature of the Abraham model. The velocity singles out a direction, which is then taken by $\omega$. Eventually one has to understand the domain of attraction of these soliton-like solutions. In this paper we want to restrict ourselves however to a somewhat simpler situation, where the charge remains at rest for all times, $q \equiv 0$. This can be achieved by assuming the (anti-) symmetry conditions

$$
E(-x)=-E(x), \quad B(-x)=B(x)
$$


for the initial fields. Then this property persists for all times. The Lorentz force equation is automatically satisfied, the Maxwell equations simplify to

$$
\dot{E}=\operatorname{rot} B-e(\omega \wedge x) \rho(x), \quad \dot{B}=-\operatorname{rot} E,
$$

and the Lorentz torque equation simplifies to

$$
I_{\mathrm{b}} \dot{\omega}=e \int x \wedge[E+(\omega \wedge x) \wedge B] \rho(x) d^{3} x .
$$

Dynamical properties of (1.8), (1.9) together with the symmetry (1.7) (= spinning charge at rest) are the subject of our paper. We consider solutions of finite energy

$$
\mathscr{H}(E, B, \omega)=\frac{I_{\mathrm{b}} \omega^{2}}{2}+\frac{1}{2} \int\left(|E(x)|^{2}+|B(x)|^{2}\right) d^{3} x<\infty .
$$

The corresponding phase space will be equipped with a suitable topology below and the existence and uniqueness of finite energy solutions will be shown.

The spinning charge at rest has a family of stationary solutions $\left(E_{\omega}, B_{\omega}\right)$ labelled by $\omega \in \mathbb{R}^{3}$. In the Fourier representation the stationary solutions are of the form, cf. [11],

$$
\hat{E}_{\omega}=-i \frac{k \hat{\rho}(k)}{k^{2}}, \quad \hat{\boldsymbol{B}}_{\omega}=-\frac{\left(k \wedge\left(\omega \wedge \nabla_{k}\right)\right) \hat{\rho}(k)}{k^{2}} .
$$

Below by "solitons" we mean these stationary solutions. The system (1.8), (1.9) with constraints (1.2), (1.7) admits no other stationary solutions. Thus it is natural to ask whether

$$
\omega(t) \rightarrow \omega_{ \pm} \quad \text { as } \quad t \rightarrow \pm \infty,
$$

to estimate the rate of convergence, and to also establish corresponding asymptotics of the field. We will resolve this questions under rather weak conditions on the initial data. Since the contraction method developed in $[5,8]$ is used, $|e|$ has to be sufficiently small, however.

Physically, an external forcing of the spin is most easily supplemented by a uniform external magnetic field $B_{\mathrm{ex}}=\varepsilon \bar{B}$, with $\bar{B}$ a fixed vector and $\varepsilon$ controlling the strength of the field. The Lorentz torque equation reads then

$$
I_{\mathrm{b}} \dot{\boldsymbol{\omega}}=e \int x \wedge[E+(\omega \wedge x) \wedge(B+\varepsilon \overline{\boldsymbol{B}})] \rho(x) d^{3} x
$$

that can be transformed to

$$
\dot{\omega}=\frac{e}{2 m} \omega \wedge \varepsilon \bar{B}+\frac{e}{I_{\mathrm{b}}} \int x \wedge[E+(\omega \wedge x) \wedge B] \rho(x) d^{3} x .
$$

If the coupling to the Maxwell field is ignored, then according to (1.13) one has

$$
\dot{\omega}=\frac{e}{2 m} \omega \wedge \varepsilon \bar{B} .
$$


For non-zero coupling and small $\varepsilon \bar{B}$, the Maxwell field generates an extra torque which maintains precession but modifies its frequency according to

$$
\dot{\omega}=\frac{e g}{2 m} \omega \wedge \varepsilon \bar{B}
$$

which defines the gyromagnetic ratio $g$. We will show that on the time scale $t=\varepsilon^{-1} \tau, \tau=\mathcal{O}(1),(1.15)$ is a good approximation to the true dynamics and we identify the gyromagnetic ratio as

$$
g=\frac{I_{\mathrm{b}}}{I_{\mathrm{b}}+I_{\mathrm{f}}}
$$

where

$$
I_{\mathrm{f}}=\frac{2}{3} e^{2} \int\left|\nabla_{k} \hat{\rho}(k)\right|^{2}|k|^{-2} d^{3} k
$$

is the field moment of inertia generated by the coupling through the Maxwell field. At higher corrections to the effective dynamics one expects to have radiative damping. We refer to [11], Chapter 10, for a more exhaustive discussion of the damping. The convergence (1.12) for the soliton with $q \equiv 0$ is discussed also in [2] for axially symmetric case.

\section{Main Results}

As in any dynamical problem, the first step is to define a suitable phase space. Let $L^{2}$ denote the real Hilbert space $L^{2}\left(\mathbb{R}^{3}, \mathbb{R}^{3}\right)$ with the norm $|\cdot|$ and the scalar product $\langle\cdot, \cdot\rangle$. We introduce the Hilbert spaces $\mathscr{F}=L^{2} \oplus L^{2}$ and $\mathscr{L}=\mathscr{F} \oplus \mathbb{R}^{3}$ with finite norms

$$
\begin{gathered}
\|(E(x), B(x))\|_{\mathscr{F}}=|E|+|B|, \\
\|Y\|_{\mathscr{L}}=|E|+|B|+|\omega| \quad \text { for } Y=(E(x), B(x), \omega) \in \mathscr{L} .
\end{gathered}
$$

$\mathscr{L}$ is the space of finite energy states. The energy functional (1.10) is continuous in the space $\mathscr{L}$. In $\mathscr{F}$ and $\mathscr{L}$ we define the local energy seminorms by

$$
\begin{gathered}
\|(E(x), B(x))\|_{R}=|E|_{R}+|B|_{R}, \\
\|Y\|_{R}=|E|_{R}+|B|_{R}+|\omega| \quad \text { for } Y=(E(x), B(x), \omega)
\end{gathered}
$$

for every $R>0$, where $|\cdot|_{R}$ is the norm in $L^{2}\left(B_{R}\right), B_{R}$ is the ball $\left\{x \in \mathbb{R}^{3}:|x|<R\right\}$. Let us denote by $\mathscr{F}_{F}, \mathscr{L}_{F}$ the spaces $\mathscr{F}, \mathscr{L}$ equipped with the Fréchet topology induced by these seminorms. Note that the spaces $\mathscr{L}$ and $\mathscr{L}_{F}, \mathscr{F}_{F}$ are metrisable, but $\mathscr{L}_{F}, \mathscr{F}_{F}$ are not complete.

The system (1.1) has to satisfy the constraints (1.2). Therefore the actual phase space is a nonlinear submanifold of the linear space $\mathscr{L}$.

Definition 2.1. (i) The phase space $\mathscr{M}$ for Maxwell-Lorentz equations including spin (1.1) is the metric space of states $(E(x), B(x), \omega) \in \mathscr{L}$ satisfying (1.7) and the constraints,

$$
\operatorname{div} E(x)=e \rho(x) \quad \text { and } \quad \operatorname{div} B(x)=0 \quad \text { for } x \in \mathbb{R}^{3} .
$$

The metric on $\mathscr{M}$ is induced through the embedding $\mathscr{M} \subset \mathscr{L}$. 
(ii) $\mathscr{M}^{\sigma}$ for $0 \leqslant \sigma \leqslant 1$ is the set of the states $(E(x), B(x), \omega) \in \mathscr{M}$ such that $\nabla E(x), \nabla B(x)$ are $L_{\mathrm{loc}}^{\infty}$ outside the ball $B_{R}$ with some $R=R(Y)>0$ and

$$
|E(x)|+|B(x)|+|x|(|\nabla E(x)|+|\nabla B(x)|) \leqslant C|x|^{-1-\sigma} \text { for }|x|>R \text {. }
$$

Let us write the system (1.2), (1.8), (1.13) as a dynamical equation in $\mathscr{M}$

$$
\dot{Y}(t)=\mathbf{F}_{\varepsilon}(Y(t)), \quad t \in \mathbb{R},
$$

where $Y(t)=(E(x, t), B(x, t), \omega(t)) \in \mathscr{M}$. Then

Proposition 2.2. Let $(C)$ hold, $\varepsilon \in \mathbb{R}$, and let $Y^{0}=\left(E^{0}(x), B^{0}(x), \omega^{0}\right) \in \mathscr{M}$.

(i) the system (2.5) has a unique solution $Y(t)=(E(x, t), B(x, t)$, $\omega(t)) \in C(\mathbb{R}, \mathscr{M})$ with $Y(0)=Y^{0}$;

(ii) the energy is conserved, i.e.,

$$
\mathscr{H}(Y(t))=\mathscr{H}\left(Y^{0}\right), \quad t \in \mathbb{R} .
$$

The proof is similar to that of [6], Proposition 2.3.

Denote the field part of the solution by $F(x, t)=(E(x, t), B(x, t))$. Let $F_{\omega}(x)=$ $\left(E_{\omega}(x), B_{\omega}(x)\right)$ be the field component of a solution of angular velocity $\omega$. Let $U(t)$ be the group of the free Maxwell-Lorentz equations, cf. section 3. First consider the "unperturbed" system (2.5) with $\varepsilon=0$.

Theorem 2.3. Let the conditions of Proposition 2.2 hold. Let $|e|<e_{0}$ with sufficiently small $e_{0}=e_{0}\left(R_{\rho}\right), Y(0) \in \mathscr{M}^{\sigma}$ with some $\sigma \in(0,1]$. Consider the solution

$$
Y(t)=(E(x, t), B(x, t), \omega(t)) \in C(\mathbb{R}, \mathscr{M})
$$

to (2.5) with $\varepsilon=0$. Then $Y(t)$ admits the following long-time asymptotics:

(i) There exist $\omega_{ \pm}=\lim _{t \rightarrow \pm \infty} \omega(t)$ such that

$$
\left|\omega(t)-\omega_{ \pm}\right| \leqslant C(1+|t|)^{-\sigma}
$$

and the fields converge in the local energy seminorms:

$$
\left\|F(x, t)-F_{\omega_{ \pm}}(x)\right\|_{R} \leqslant C_{R}(1+|t|)^{-\sigma}, \quad \forall R>0 .
$$

(ii) There exist $F_{ \pm} \in \mathscr{F}$ such that the fields admit the following "scattering" asymptotics in the global energy norm:

$$
\left\|F(x, t)-F_{\omega(t)}(x)-U(t) F_{ \pm}\right\|_{\mathscr{F}} \leqslant C(1+|t|)^{-\sigma} .
$$

Next consider the "perturbed" system (2.5) with an arbitrary $\varepsilon \in \mathbb{R}$.

Theorem 2.4. Let the conditions of Theorem (2.3) hold. Consider the solution $Y(t)=(E(x, t), B(x, t), \omega(t)) \in C(\mathbb{R}, \mathscr{M})$ to (2.5) with $\varepsilon \in \mathbb{R}$, and let $F(0)=F_{\omega(0)}$. Then the following uniform in $t \in \mathbb{R}$ bounds hold:

$$
\begin{gathered}
\left\|F(x, t)-F_{\omega(t)}(x, t)\right\|_{R} \leqslant C_{R} \varepsilon, \quad R>0, \\
|\dot{\omega}(t)| \leqslant C \varepsilon
\end{gathered}
$$




$$
|\ddot{\omega}(t)| \leqslant C \varepsilon\left(\varepsilon+\left(1+t^{2}\right)^{-1}\right) .
$$

Let us define an effective dynamics as a solution to the equation

$$
\dot{\Omega}(t)=\frac{e g}{2 m} \Omega(t) \wedge \varepsilon \bar{B},
$$

with $g$ defined by (1.16). Finally, consider the perturbed system (2.5) with small $\varepsilon$.

Theorem 2.5. Let the conditions of Theorem 2.4 hold, and $\Omega(t)$ be the solution to (2.13) with the initial condition $\Omega(0)=\omega(0)$. Then for sufficiently small $\varepsilon$ for all $\tau>0$ there exists a positive $C(\tau)$ such that

$$
|\Omega(t)-\omega(t)| \leqslant C(\tau) \varepsilon, \quad|t| \leqslant \tau \varepsilon^{-1} .
$$

Thus, for a sufficiently large interval of time the dynamics of $\omega$ can be approximated with the dynamics of the solution $\Omega$ to the finite-dimensional system (2.13). We call this asymptotics "adiabatic" in accordance with [5, 9].

\section{Convergence to Soliton and Scattering}

Let us prove Theorem 2.3. We denote

$$
Z(x, t)=F(x, t)-F_{\omega(t)}(x) .
$$

Defining $\bar{\rho}(x)=(\rho(x), 0)$ and $A(E, B)=(\operatorname{rot} B,-\operatorname{rot} E), F$ satisfies the equation of motion

$$
\dot{F}(x, t)=A F(x, t)-e(\omega(t) \wedge x) \bar{\rho}(x) .
$$

Therefore, $Z$ is governed by

$$
\dot{Z}(x, t)=A Z(x, t)-\dot{\omega}(t) \cdot \nabla_{\omega} F_{\omega(t)}(x) .
$$

Lemma 3.1. Under the assumptions of Theorem 2.3 the following bound holds for any $R>0$,

$$
\|Z(\cdot, t)\|_{R} \leqslant C_{R}(Z(\cdot, 0), \rho)(1+|t|)^{-1-\sigma}
$$

Proof. Definition (3.1) and constraints (1.2) for $F$ and $F_{\omega}$ imply $\operatorname{div} Z=0$. Therefore $Z(\cdot, t) \in \mathscr{F}_{s}=\{Z(x) \in \mathscr{F}: \operatorname{div} Z(x)=0\}$. Denote by $U(t)=\exp (A t)$ the group of the free Maxwell-Lorentz equations in $\mathscr{F}_{s}$. Namely, given $f_{0}(x)=\left(e_{0}(x), b_{0}(x)\right) \in \mathscr{F}_{s}$, then $(e(x, t), b(x, t)):=\left[U(t) f_{0}\right](x)$ reads, [9], [6, Appendix]

$$
\begin{gathered}
e(x, t)=\frac{1}{4 \pi t^{2}} \int_{|y-x|=t} d^{2} y\left[e_{0}(y)+t \nabla \wedge b_{0}(y)+((y-x) \cdot \nabla) e_{0}(y)\right], \\
b(x, t)=\frac{1}{4 \pi t^{2}} \int_{|y-x|=t} d^{2} y\left[b_{0}(y)-t \nabla \wedge e_{0}(y)+((y-x) \cdot \nabla) b_{0}(y)\right] .
\end{gathered}
$$


The action of this group is isometric on $\mathscr{F}_{s}$ according to the corresponding energy conservation law. Solving Eq. (3.3) through Duhamel method one obtains the mild solution representation

$$
\begin{aligned}
Z(t) & =U(t) Z(0)-\int_{0}^{t} U(t-s)\left[\dot{\omega}(s) \cdot \nabla_{\omega} F_{\omega(s)}(\cdot)\right] d s \\
& =U(t) Z(0)-\int_{0}^{t} \dot{\omega}(s) \cdot U(t-s) \nabla_{\omega} F_{\omega(s)}(\cdot) d s,
\end{aligned}
$$

where the set of three vectors $\nabla \omega_{j} F_{\omega}, j=1,2,3$ is denoted by $\nabla_{\omega} F_{\omega}$, and

$$
\dot{\omega} \cdot U(t-s) \nabla_{\omega} F_{\omega} \equiv \sum_{j=1}^{3} \dot{\omega}_{j} \cdot U(t-s) \nabla \omega_{j} F_{\omega} .
$$

The action of the group under the integral sign is well defined, since $\operatorname{div} \nabla \omega_{j} F_{\omega(s)}=0 . \quad$ Denote by $Z_{1}(x, t)=E(x, t)-E_{\omega(t)}(x), \quad Z_{2}(x, t)=B(x, t)-$ $B_{\omega(t)}(x)$ the components of $Z(x, t)$ and observe that

$$
\int x \wedge\left[E_{\omega}(x)+(\omega \wedge x) \wedge B_{\omega}(x)\right] \rho(x) d^{3} x=0,
$$

since the soliton (1.11) is a solution to (1.9). Then (1.9) implies

$$
\dot{\omega}(t)=\frac{e}{I_{\mathrm{b}}} \int x \wedge\left[Z_{1}(x, t)+(\omega \wedge x) \wedge Z_{2}(x, t)\right] \rho(x) d^{3} x .
$$

Thus, using $(C)$ and uniform in $t$ boundness of $\omega(t)$ that follows from the energy conservation, one obtaines

$$
|\dot{\omega}(t)| \leqslant C e\|Z(\cdot, t)\|_{R_{\rho}} .
$$

Let us denote $\bar{F}_{\omega}=\nabla_{\omega} F_{\omega}, S_{t-s}(x)=\{y:|y-x|=t-s\}$, and

$$
\bar{F}(\cdot, t, s)=U(t-s)\left[\bar{F}_{\omega(s)}(\cdot)\right] .
$$

Then [6, Appendix] implies the representation

$$
\bar{F}(x, t, s)=\sum_{|\alpha| \leqslant 1}(t-s)^{|\alpha|-2} \int_{S_{t-s}(x)} m_{\alpha}(x-y) \partial_{y}^{\alpha} \bar{F}_{\omega(s)}(y) d^{2} y
$$

because $\operatorname{div} \bar{F}_{\omega(s)}=0$. The coefficients $m_{\alpha}(\cdot)$ are bounded $6 \times 6$ matrix functions and the sum runs over the multiindices $\alpha=\left(\alpha_{1}, \alpha_{2}, \alpha_{3}\right)$ with nonnegative integers $\alpha_{j} \leqslant 1$. If $|x| \leqslant R_{\rho}$, then on the sphere $S_{t-s}(x)$

$$
|y|=|(y-x)+x| \geqslant(t-s)-|x| \geqslant(t-s)-R_{\rho} .
$$

On the other hand, the representation (1.11) yields the bounds of type (2.4) with $\sigma=1$,

$$
\left|\bar{F}_{\omega}(x)\right|+|x|\left|\nabla \bar{F}_{\omega}(x)\right| \leqslant e C\left(R_{\rho}\right)|x|^{-2}, \quad|x| \geqslant R_{\rho}+1 .
$$


Inserting (3.12) and (3.11) in the formula for $\bar{F}(x, t, s)$, we obtain the pointwise upper bound

$$
|\bar{F}(x, t, s)| \leqslant e \sum_{|\alpha| \leqslant 1}(t-s)^{|\alpha|-2} \frac{C_{1}\left(R_{\rho}\right)(t-s)^{2}}{(1+|t-s|)^{|\alpha|+2}} \leqslant e \frac{C_{2}\left(R_{\rho}\right)}{1+(t-s)^{2}}, \quad|x| \leqslant R_{\rho},
$$

provided $t-s \geqslant 3 R_{\rho}$. Therefore (3.13) implies for large $t-s$ the integral estimate

$$
\|\bar{F}(\cdot, t, s)\|_{R_{\rho}} \leqslant e \frac{C_{3}\left(R_{\rho}\right)}{1+(t-s)^{2}} .
$$

On the other hand, for bounded $t-s$ the integral estimate (3.14) follows from (3.9) because the map $U(t-s)$ is isometric, and $\left\|\nabla \omega_{j} F_{\omega}\right\|_{\mathscr{F}} \leqslant e C\left(R_{\rho}\right), j=1,2,3$ by (C) and by uniform in $t$ boundness of $\omega(t)$. Finally, (3.8) and (3.14) imply

$$
\|\dot{\omega}(s) \cdot \bar{F}(x, t, s)\|_{R_{\rho}} \leqslant e^{2} C_{4}\left(R_{\rho}\right) \frac{\|Z(\cdot, s)\|_{R_{\rho}}}{1+(t-s)^{2}} .
$$

As a last step, let us bound the first term on right hand side of (3.6). Note that we have derived (3.14) from the bound (3.12) which corresponds to (2.4) with $\sigma=1$. Since $Y(0) \in \mathscr{M}^{\sigma}$ by assumption, $F(x, 0)=(E(x, 0), B(x, 0))$ satisfies the bound (2.4) with a certain $\sigma \in(0,1]$. On the other hand, $F_{\omega(0)}(x)$ satisfies the bound (2.4) with $\sigma=1$. Hence, $Z(x, 0)=F(x, 0)-F_{\omega(0)}(x)$ satisfies (2.4) with the same $\sigma$, as $F(x, 0)$. Therefore, proceeding as in (3.12)-(3.14), one gets

$$
\|U(t) Z(0)\|_{R} \leqslant C(R)(1+|t|)^{-1-\sigma} \text {. }
$$

Combining (3.6) to (3.15) and (3.16) we arrive at

$$
\|Z(\cdot, t)\|_{R_{\rho}} \leqslant \frac{C\left(R_{\rho}\right)}{(1+|t|)^{1+\sigma}}+e^{2} C_{4}\left(R_{\rho}\right) \int_{0}^{t} \frac{\|Z(\cdot, s)\|_{R_{\rho}}}{1+(t-s)^{2}} d s, \quad t \geqslant 0 .
$$

Thus, setting $M(t)=\max _{0 \leqslant s \leqslant t}(1+|s|)^{1+\sigma}\|Z(x, s)\|_{R_{\rho}}$, we have

$$
M(t) \leqslant C_{0}+e^{2} C(\rho) I_{\sigma} M(t),
$$

where

$$
I_{\sigma}=\sup _{t \geqslant 0}(1+|t|)^{1+\sigma} \int_{0}^{t} \frac{(1+|s|)^{-1-\sigma}}{(1+|t-s|)^{2}} d s<\infty \quad \text { for } \sigma \in(0,1] .
$$

It remains to choose $e^{2} C(\rho) I_{\sigma}<1$, then (3.4) with $R=R_{\rho}$ follows. We claim that the bound (3.4) with $R=R_{\rho}$ implies (3.4) for any $R>0$. Indeed, (3.14)-(3.17) hold with the norm $\|\cdot\|_{R}$ instead of $\|\cdot\|_{R_{\rho}}$ on the left hand sides and with $C_{i}(R)$ instead of $C_{i}\left(R_{\rho}\right)$ on the right hand sides. Then (3.17) with this generalization and (3.4) with $R=R_{\rho}$ imply (3.4) for any $R>0$.

Proof of Theorem 2.3. (i) (3.4) with $R=R_{\rho}$ and (3.7) imply

$$
|\dot{\omega}(t)| \leqslant C(1+|t|)^{-1-\sigma}
$$

Therefore the limits $\omega_{ \pm}$exist and (2.7) follows. By (3.4) also (2.8) holds. 
(ii) We have to prove that $\left\|Z(x, t)-U(t) F_{ \pm}\right\|_{\mathscr{F}} \leqslant C(1+|t|)^{-\sigma}$. This is equivalent to $\left\|U(-t) Z(x, t)-F_{ \pm}\right\|_{\mathscr{F}} \leqslant C(1+|t|)^{-\sigma}$ since the group $U(t)$ is isometric. Apply $U(-t)$ to integral Eq. (3.6) and get $U(-t) Z(t)=$ $Z(0)-\int_{0}^{t} U(-s)\left[\dot{\omega}(s) \cdot \nabla_{\omega} F_{\omega(s)}(\cdot)\right] d s$. Then (3.18) implies the convergence of the integral with the mentioned rate.

Theorem 2.3 is proved.

\section{A Priori Bounds}

Here we prove Theorem 2.4. First, the proof of (2.10) repeats with some modifications the one of Theorem 2.3. The bound (3.8) now reads

$$
|\dot{\omega}(t)| \leqslant C e\left(\|Z(\cdot, t)\|_{R_{\rho}}+\varepsilon\right) .
$$

Instead of (3.15) we obtain

$$
\|\dot{\omega}(s) \cdot \bar{F}(x, t, s)\|_{R_{\rho}} \leqslant e^{2} C_{4}\left(R_{\rho}\right) \frac{\|Z(\cdot, s)\|_{R_{\rho}}+\varepsilon}{1+(t-s)^{2}}
$$

Since $Z(0)=0$ and $U(t) Z(0)=0$, the estimate (3.17) becomes

$$
\|Z(\cdot, t)\|_{R_{\rho}} \leqslant e^{2} C_{4}\left(R_{\rho}\right) \int_{0}^{t} \frac{\|Z(\cdot, s)\|_{R_{\rho}}+\varepsilon}{1+(t-s)^{2}} d s, \quad t \geqslant 0 .
$$

We set $M(t)=\max _{0 \leqslant s \leqslant t}\|Z(x, s)\|_{R_{\rho}}$ and obtain

$$
M(t) \leqslant e^{2} C(\rho) I(M(t)+\varepsilon),
$$

where

$$
I=\sup _{t \geqslant 0} \int_{0}^{t} \frac{1}{(1+|t-s|)^{2}} d s<\infty .
$$

Finally choose $e^{2} C(\rho) I<1$, then (2.10) with $R=R_{\rho}$ follows. The rest of the proof of (2.10) is the same as the one of Theorem 2.3.

Next let us prove (2.11). The angular velocity $\omega$ obeys the following equation of motion,

$$
\dot{\omega}=\frac{e}{2 m} \omega \wedge \varepsilon \bar{B}+\frac{e}{I_{\mathrm{b}}} \int x \wedge[E+(\omega \wedge x) \wedge B] \rho(x) d^{3} x .
$$

On the other hand, for the corresponding soliton we have

$$
0=\frac{e}{I_{\mathrm{b}}} \int x \wedge\left[E_{\omega}+(\omega \wedge x) \wedge B_{\omega}\right] \rho(x) d^{3} x .
$$

So, by subtraction

$$
\dot{\omega}=\frac{e}{2 m} \omega \wedge \varepsilon \bar{B}+\frac{e}{I_{\mathrm{b}}} \int x \wedge\left[Z_{1}+(\omega \wedge x) \wedge Z_{2}\right] \rho(x) d^{3} x .
$$


Recall that by the energy conservation law (2.6) $|\omega(t)|$ is bounded uniformly in $t \in \mathbb{R}$. (2.11) follows then from (4.4) and (2.10).

It remains to prove (2.12). We differentiate (4.4) in $t$ and obtain

$$
\begin{aligned}
\ddot{\omega}= & \frac{e}{2 m} \dot{\omega} \wedge \varepsilon \bar{B}+\frac{e}{I_{\mathrm{b}}} \int x \wedge\left[\dot{Z}_{1}+(\dot{\omega} \wedge x) \wedge Z_{2}+(\omega \wedge x) \wedge \dot{Z}_{2}\right] \rho(x) d^{3} x \\
= & \frac{e}{2 m} \dot{\omega} \wedge \varepsilon \bar{B}+\frac{e}{I_{\mathrm{b}}} \int x \wedge\left[(\dot{\omega} \wedge x) \wedge Z_{2}\right] \rho(x) d^{3} x \\
& +\frac{e}{I_{\mathrm{b}}} \int x \wedge\left[\dot{Z}_{1}+(\omega \wedge x) \wedge \dot{Z}_{2}\right] \rho(x) d^{3} x .
\end{aligned}
$$

From the estimates (2.11) and (2.10) it follows that

$$
|\ddot{\omega}(t)| \leqslant C\left(\varepsilon^{2}+|M(t)|\right),
$$

with

$$
M(t)=\langle G(t), \bar{\rho}\rangle
$$

where

$$
G(t)=\left(\begin{array}{c}
x \wedge \dot{Z}_{1} \\
x \wedge\left((\omega \wedge x) \wedge \dot{Z}_{2}\right)
\end{array}\right), \quad \bar{\rho}(x)=\left(\begin{array}{c}
\rho(x) \\
\rho(x)
\end{array}\right) .
$$

Therefore (2.12) is a consequence of

Lemma 4.1. Let e be sufficiently small. Then for $t \in \mathbb{R}$ the bound holds

$$
|M(t)| \leqslant C\left(R_{\rho}\right) \varepsilon\left(\varepsilon+\left(1+t^{2}\right)^{-1}\right) .
$$

Proof. We extend the method of the previous section. Denote $\Xi(x, t)=\dot{Z}(x, t)$. To obtain an equation for $\Xi(t)$ we differentiate Eq. (3.3) in $t$ and arrive at

$$
\dot{\Xi}(x, t)=A \Xi(x, t)-\ddot{\omega} \cdot \nabla_{\omega} F_{\omega}-\left(\dot{\omega} \cdot \nabla_{\omega}\right)^{2} F_{\omega} .
$$

Thus,

$$
\Xi(t)=U(t) \Xi(0)-\int_{0}^{t} U(t-s)\left[\ddot{\omega} \cdot \nabla_{\omega} F_{\omega}(s)+\left(\dot{\omega} \cdot \nabla_{\omega}\right)^{2} F_{\omega}(s)\right] d s .
$$

For $M(t)$ we have

$$
M(t)=\left\langle G_{1}(t), \bar{\rho}\right\rangle-\left\langle G_{2}(t), \bar{\rho}\right\rangle-\left\langle G_{3}(t), \bar{\rho}\right\rangle,
$$

where

$$
\begin{gathered}
G_{1}(t)=\left(\begin{array}{c}
x \wedge[U(t) \dot{Z}(0)]_{1} \\
x \wedge\left((\omega \wedge x) \wedge[U(t) \dot{Z}(0)]_{2}\right)
\end{array}\right), \\
G_{2}(t)=\left(\begin{array}{c}
x \wedge\left[\int_{0}^{t} U(t-s) \ddot{\omega} \cdot \nabla_{\omega} F_{\omega} d s\right]_{1} \\
x \wedge\left((\omega \wedge x) \wedge\left[\int_{0}^{t} U(t-s) \ddot{\omega} \cdot \nabla_{\omega} F_{\omega} d s\right]_{2}\right)
\end{array}\right),
\end{gathered}
$$




$$
G_{3}(t)=\left(\begin{array}{c}
x \wedge\left[\int_{0}^{t} U(t-s)\left(\dot{\omega} \cdot \nabla_{\omega}\right)^{2} F_{\omega} d s\right]_{1} \\
x \wedge\left((\omega \wedge x) \wedge\left[\int_{0}^{t} U(t-s)\left(\dot{\omega} \cdot \nabla_{\omega}\right)^{2} F_{\omega} d s\right]_{2}\right)
\end{array}\right)
$$

We estimate the three summands one by one.

(i) For the first summand one notes that $\dot{Z}(0)=-\left.\dot{\omega}(t) \cdot \nabla_{\omega} F_{\omega(t)}(x)\right|_{t=0}$ because of (3.3) and since $Z(0)=0$. Recall that $F_{\omega}=\left(E_{\omega}, B_{\omega}\right)$, where $\hat{E}_{\omega}$ and $\hat{\boldsymbol{B}}_{\omega}$ are given by (1.11). Since $E_{\omega}$ does not depend on $\omega, \dot{Z}_{1}(0)=0$. Furthermore, it is easy to obtain that $B_{\omega}(x)=\mathcal{O}\left(|x|^{-2}\right)$ as $|x| \rightarrow \infty$, hence $\dot{Z}_{2}(0)=\mathcal{O}\left(|x|^{-2}\right)$ as $|x| \rightarrow \infty$. Apply $U(t)$ to $\left(0, \dot{Z}_{2}(0)\right)$, see $(3.5)$, recall that $\dot{\omega}(0)=\frac{e}{2 m} \omega(0) \wedge \varepsilon \bar{B}$, and obtain that $U(t) \dot{Z}(0)=\mathcal{O}\left(\varepsilon t^{-2}\right)$ as $t \rightarrow \infty$.

More precisely, by the energy conservation for the free Maxwell-Lorentz equations, one obtains that $\|U(t) \dot{Z}(0)\|_{\mathscr{F}} \leqslant \varepsilon \cdot \min \left(C_{1}, C_{2} t^{-2}\right)$ with $C_{1}, C_{2}$ depending on initial data and $|\bar{B}|$. Hence,

$$
\left|\left\langle G_{1}(t), \bar{\rho}\right\rangle\right| \leqslant \varepsilon \cdot \min \left(C_{1}^{\prime}, C_{2}^{\prime} t^{-2}\right)
$$

with $C_{1}^{\prime}, C_{2}^{\prime}$ depending on initial data, $|\bar{B}|$ and $R_{\rho}$. Below we suppress the dependence on initial data and $|\bar{B}|$.

(ii) From (4.5) and (2.11), (2.10) it follows that $\left(\ddot{\omega} \cdot \nabla_{\omega} F_{\omega}\right)(x, t)=$ $e(x, t)+m(x, t)$, where by $(C)$ again $(\mathrm{cf}(4.6))$

$$
\sup _{t \geqslant 0}\|e(x, t)\|_{\mathscr{F}} \leqslant \varepsilon^{2} C\left(R_{\rho}\right) e, \quad\|m(x, t)\|_{\mathscr{F}} \leqslant C\left(R_{\rho}\right) e|M(t)| .
$$

Therefore by repeating the argument in (3.9) to (3.15) we arrive at the estimate

$$
\left|\left\langle G_{2}(t), \bar{\rho}\right\rangle\right| \leqslant C_{2}\left(R_{\rho}\right) e^{2} \int_{0}^{t} \frac{\varepsilon^{2}+|M(s)|}{1+(t-s)^{2}} d s, \quad t \geqslant 0 .
$$

(iii) For the third summand in (4.9) the estimate

$$
\left|\left\langle G_{3}(t), \bar{\rho}\right\rangle\right| \leqslant \varepsilon^{2} C\left(R_{\rho}\right)
$$

follows from (2.11), (C), and properties of the group $U(t)$.

Finally, one substitutes (4.13), (4.14), (4.15), and (4.16) to (4.9) and obtains the integral inequality

$$
|M(t)| \leqslant \varepsilon \cdot \min \left(C_{1}^{\prime}\left(R_{\rho}\right), C_{2}^{\prime}\left(R_{\rho}\right) t^{-2}\right)+C_{3}\left(R_{\rho}\right) \varepsilon^{2}+C_{4}\left(R_{\rho}\right) e^{2} \int_{0}^{t} \frac{\varepsilon^{2}+|M(s)|}{1+(t-s)^{2}} d s .
$$

Therefore (4.7) follows provided that $e \leqslant e_{0}\left(R_{\rho}\right)$.

\section{Self-torque}

To prove Theorem 2.5 we analyse the self-torque

$$
T_{\text {self }}(t)=\int x \wedge[E+(\omega \wedge x) \wedge B] \rho(x) d^{3} x .
$$

We represent the fields as the sum

$$
E=E_{(0)}+E_{(\mathrm{r})}, \quad B=B_{(0)}+B_{(\mathrm{r})},
$$


where $E_{(0)}, B_{(0)}$ are initial fields propagated to the time $t$, and $E_{(\mathrm{r})}, B_{(\mathrm{r})}$ are retarded fields. In $[11,9,6]$ both $x$ - and $k$-representations for $E_{(0)}, B_{(0)}, E_{(\mathrm{r})}, B_{(\mathrm{r})}$ are given. Then we obtain

$$
\begin{aligned}
T_{\text {self }}(t)= & \int x \wedge\left[E_{(0)}+(\omega \wedge x) \wedge B_{(0)}\right] \rho(x) d^{3} x \\
& +\int x \wedge\left[E_{(\mathrm{r})}+(\omega \wedge x) \wedge B_{(\mathrm{r})}\right] \rho(x) d^{3} x \\
= & T_{(0)}(t)+T_{(\mathrm{r})}(t) .
\end{aligned}
$$

Proposition 5.1. Let $I_{\mathrm{f}}$ be defined by (1.17). Then

(i) $T_{(0)}(t)=0$ for $t>2 R_{\rho}$ and

(ii) for $t \in \mathbb{R}$

$$
T_{(\mathrm{r})}(t)=-I_{\mathrm{f}} \dot{\omega}(t)+\delta(\varepsilon, t)
$$

with $|\delta(\varepsilon, t)| \leqslant C \varepsilon\left(\varepsilon+\left(1+t^{2}\right)^{-1}\right)$.

Proof. (i) The fields $E_{(0)}$ and $B_{(0)}$ are the initial fields propagated to the time $t$. Similar to [9], Lemma 4.1, one obtains

$$
\left(\begin{array}{c}
E_{(0)}(x, t) \\
\dot{E}_{(0)}(x, t)
\end{array}\right)=e \int_{-\infty}^{0} d s G(t-s)\left(\begin{array}{c}
-\left(\omega_{0} \wedge \cdot\right) \rho(\cdot) \\
-\nabla \rho(\cdot)
\end{array}\right)[x],
$$

where $G(t)$ is the group generated by the free wave equation. Since $\rho$ has its support in $\bar{B}_{R_{\rho}}(0)$, one has $E_{(0)}(x, t)=0$ for $t>|x|+R_{\rho}$. Then the term $\int\left(x \wedge E_{(0)}\right) \rho(x) d^{3} x$ of $T_{(0)}(t)$ is equal to zero for $t>2 R_{\rho}$. For the term of $T_{(0)}(t)$ containing $B_{(0)}(x, t)$ the argument is similar.

(ii) For $T_{(\mathrm{r})}(t)$ we use Fourier transform to have

$$
T_{(\mathrm{r})}(t)=-\int i \nabla_{k} \hat{\rho}(k) \wedge \hat{E}_{(\mathrm{r})} d^{3} k-\int i \nabla_{k} \hat{\rho}(k) \wedge\left[\left(\omega \wedge i \nabla_{k}\right) \wedge \hat{\boldsymbol{B}}_{(\mathrm{r})}\right] d^{3} k .
$$

By substitution, compare with [11,9], denoting $\hat{k}=k /|k|$,

$$
\begin{aligned}
& \hat{E}_{(\mathrm{r})}(t)=-\int_{0}^{t} d s \cos (|k|(t-s)) \omega(s) \wedge i \nabla_{k} \hat{\rho}(k)+i \int_{0}^{t} d s \sin (|k|(t-s)) \hat{k} \hat{\rho}(k), \\
& \hat{B}_{(\mathrm{r})}(t)=-i \int_{0}^{t} d s \sin (|k|(t-s)) \hat{k} \wedge\left(\omega(s) \wedge i \nabla_{k}\right) \hat{\rho}(k)
\end{aligned}
$$

we obtain

$$
\begin{aligned}
T_{(\mathrm{r})}(t)= & -i \int d^{3} k \nabla_{k} \hat{\rho}(k) \wedge\left(-\int_{0}^{t} d s \cos (|k|(t-s)) \omega(s) \wedge i \nabla_{k} \hat{\rho}(k)\right) \\
& +\int d^{3} k \nabla_{k} \hat{\rho}(k) \wedge\left(\int_{0}^{t} d s \sin (|k|(t-s)) \hat{k} \hat{\rho}(k)\right) \\
& -i \int d^{3} k i \nabla_{k} \wedge\left[\left(\omega \wedge i \nabla_{k}\right) \wedge i \int_{0}^{t} d s \sin (|k|(t-s)) \hat{k} \wedge\left(\omega(s) \wedge i \nabla_{k}\right) \hat{\rho}(k)\right] \hat{\rho}(k) .
\end{aligned}
$$


The second integral is zero, since $\nabla_{k} \hat{\rho}(k)$ is collinear to $k$. The third integral simplifies to

$$
\int d^{3} k|k|^{-1} \nabla_{k} \hat{\rho} \wedge\left[\nabla_{k} \hat{\rho} \wedge\left(\omega(t) \wedge \int_{0}^{t} d s \sin (|k|(t-s)) \omega(s)\right)\right] .
$$

Finally,

$$
\begin{aligned}
T_{(\mathrm{r})}(t)= & -\int d^{3} k \int_{0}^{t} d s \cos (|k|(t-s)) \nabla_{k} \hat{\rho} \wedge\left(\omega(s) \wedge \nabla_{k} \hat{\rho}\right) \\
& -\int d^{3} k|k|^{-1} \nabla_{k} \hat{\rho} \wedge\left[\nabla_{k} \hat{\rho} \wedge\left(\omega(t) \wedge \int_{0}^{t} d s \sin (|k|(t-s)) \omega(s)\right)\right] .
\end{aligned}
$$

Returning to the $x$-representation, one obtains that, provided $t, T>2 R_{\rho}$, the integrals $\int_{0}^{t}$ can be replaced by $\int_{t-T}^{t}$ and do not depend on $T$, compare with [5, 9]. Let us complete the computation of the $T_{(\mathrm{r})}(t)$. We use the Taylor expansion $\omega(s)=\omega(t)-\dot{\omega}(t)(t-s)+\alpha(\varepsilon, t)$ with $|\alpha(\varepsilon, t)| \leqslant C \varepsilon\left(\varepsilon+\left(1+t^{2}\right)^{-1}\right)$ according to (2.12). Then for the first integral of (5.2) one obtains

$$
\begin{aligned}
-\int d^{3} k & \int_{t-T}^{t} d s \cos (|k|(t-s)) \nabla_{k} \hat{\rho} \wedge\left[(\omega(t)-\dot{\omega}(t)(t-s)+\alpha(\varepsilon, t)) \wedge \nabla_{k} \hat{\rho}\right] \\
= & -\int d^{3} k|k|^{-1} \sin |k| T \nabla_{k} \hat{\rho} \wedge\left(\omega(t) \wedge \nabla_{k} \hat{\rho}\right) \\
& +T \int d^{3} k\left(|k|^{-1} \sin |k| T+|k|^{-2} \cos |k| T\right) \nabla_{k} \hat{\rho} \wedge\left(\dot{\omega}(t) \wedge \nabla_{k} \hat{\rho}\right) \\
& -\int d^{3} k|k|^{-2} \nabla_{k} \hat{\rho} \wedge\left(\dot{\omega}(t) \wedge \nabla_{k} \hat{\rho}\right)+\beta(\varepsilon, t)
\end{aligned}
$$

and for the second integral of (5.2)

$$
\begin{aligned}
& \int d^{3} k|k|^{-1} \nabla_{k} \hat{\rho} \wedge {\left[\nabla_{k} \hat{\rho} \wedge\left(\omega(t) \wedge \int_{t-T}^{t} d s \sin (|k|(t-s))(\omega(t)-\dot{\omega}(t)(t-s)+\alpha(\varepsilon, t))\right]\right.} \\
&= \int d^{3} k|k|^{-1} \nabla_{k} \hat{\rho} \wedge\left[\nabla_{k} \hat{\rho} \wedge\left(\omega(t) \wedge \omega(t) \frac{1-\cos |k| T}{|k|}\right)\right] \\
&+\int d^{3} k|k|^{-1} \nabla_{k} \hat{\rho} \wedge\left[\nabla_{k} \hat{\rho} \wedge\left(\omega(t) \wedge \dot{\omega}(t) \frac{|k| T \cos |k| T-\sin |k| T}{|k|^{2}}\right)\right]+\gamma(\varepsilon, t) .
\end{aligned}
$$

The first integral in (5.4) equals zero, the first and the second integral in (5.3), and the second integral in (5.4) are oscillatory and tend to zero as $T \rightarrow \infty$. The sum of all integrals in (5.3) and (5.4) does not depend on $T$ and hence equals its limit as $T \rightarrow \infty$. Finally,

$$
\begin{aligned}
T_{(\mathrm{r})}(t) & =-\int d^{3} k|k|^{-2} \nabla_{k} \hat{\rho} \wedge\left(\dot{\omega}(t) \wedge \nabla_{k} \hat{\rho}\right)+\delta(\varepsilon, t) \\
& =-\frac{2}{3} \dot{\omega}(t) \int d^{3} k|k|^{-2}\left|\nabla_{k} \hat{\rho}\right|^{2}+\delta(\varepsilon, t)
\end{aligned}
$$

with $|\delta(\varepsilon, t)| \leqslant C^{\prime} \varepsilon\left(\varepsilon+\left(1+t^{2}\right)^{-1}\right)$. The statement (ii) is proved. 


\section{Effective Dynamics}

We complete the proof of Theorem 2.5. Recall the effective equation

$$
\dot{\Omega}=\frac{e g}{2 m} \Omega \wedge \varepsilon \bar{B},
$$

where $g$ is given by (1.16). Let $\Omega$ be the solution to (6.1) with the initial condition $\Omega(0)=\omega(0)$. Set $\mu=e g / 2 m$. Then $r \equiv \omega-\Omega$ obeys the equation

$$
\dot{r}=\varepsilon \mu r \wedge \bar{B}+\alpha_{\varepsilon}(t)
$$

with the initial condition $r(0)=0$. Here $\left|\alpha_{\varepsilon}(t)\right| \leqslant C \varepsilon\left(\varepsilon+\left(1+t^{2}\right)^{-1}\right)$. For bounded $|t|$ this follows from the Eq. (4.4), for large $|t|$ from Proposition 5.1. Resolving the linear inhomogeneous Eq. (6.2), one obtains the following estimate for $|r(t)|$,

$$
|r(t)| \leqslant C_{1} \int_{0}^{t}\left|\alpha_{\varepsilon}(s)\right| d s .
$$

This implies (2.14). Theorem 2.5 is proved.

\section{References}

[1] Appel W, Kiessling MK-H (2001) Mass and spin renormalization in Lorentz electrodynamics. Annals of Phys 289: 24-83

[2] Appel W, Kiessling MK-H (2002) Scattering and radiation damping in gyroscopic Lorentz electrodynamics. Letters Math Phys 59: 31-46

[3] Bauer G, Dürr D (2001) The Maxwell-Lorentz system of a rigid charge distribution. Annals Inst H Poincaré 2: 179-196

[4] Kiessling M (1999) Classical electron theory and conservation laws. Comm Phys Lett A 258: 197-204

[5] Komech A, Kunze M, Spohn H (1999) Effective dynamics for a mechanical particle coupled to a wave field. Comm Math Phys 203: 1-19

[6] Komech A, Spohn H (2000) Long-time asymptotics for the coupled Maxwell-Lorentz equations. Comm PDE 25: 558-585

[7] Komech A, Spohn H (1998) Soliton-like asymptotics for a classical particle interacting with a scalar wave field. Nonlin Analysis 33: 13-24

[8] Komech A, Spohn H, Imaikin V (2000) Soliton-like asymptotics and scattering for coupled Maxwell-Lorentz equations. In: Bermúder A et al (eds) Math and Num aspects of waves propagation pp 329-333. Philadelphia, PA: SIAM

[9] Kunze M, Spohn H (2000) Adiabatic limit for the Maxwell-Lorentz equation. Ann Henri Poincaré 1: $625-653$

[10] Schwarzschild K (1903) Zur Elektrodynamik III. Über die Bewegung des Electrons. Göttinger Nachrichten: 245-278

[11] Spohn H (2002) Dynamics of charged particles and their radiation field. Preprint TU-Muenchen

Authors' addresses: V. Imaikin, Institute of Mathematics, University of Vienna, Nordbergstrasse 15, 1090 Vienna, Austria, e-mail: vimaikin@mat.univie.ac.at; A. Komech, Institute of Mathematics, University of Vienna, Nordbergstrasse 15, 1090 Vienna, Austria, e-mail: komech@mathematik.tu-muenchen.de; H. Spohn, Zentrum Mathematik, TU München, 80290 München, Germany, e-mail: spohn@mathematik. tu-muenchen.de 